\section{PWE-092 INTERNAL CONSISTENCIES OF MODERN REFLUX MONITORING METHODS: PH-IMPEDANCE MONITORING VS BRAVO PH MONITORING}

Ismail Miah*. Guy's and St Thomas' NHS Trust, Greater London, UK

\subsection{6/gutjnl-2019-BSGAbstracts.412}

Introduction There are no established guidelines for the choice of reflux monitoring equipment. Neither are we any wiser of the accuracy and consistency of the reflux monitoring techniques. This study addresses the recording consistencies of the multichannel $\mathrm{pH}$-impedance (MII-pH) technique and the wireless Bravo $\mathrm{pH}$ (WB-pH) method.

Method Patients who had 2 days of reflux monitoring using either MII-pH or WB-pH procedures in the year 2018 were selected. The consistency of normal and pathological reflux were compared between both days. The normal range considered for MII-pH was considered when total exposure was $<4.3 \%$ and DeMeester $<14.72$. WB-pH monitoring was based on the following recording for day 1 and day 2 [1].

Inter-rater reliability (IRR) was employed to assess the correlation for days 1 and 2 for each method and fisher exact test was used to assess for statistical significance in consistencies between methods. Statistical significance using fisher exact was also employed to assess diagnostic yield by extending the MII-pH to day 2 and the necessity to have extended WB-pH monitoring. We also compared the technical failure of capturing data in each method.

Results Total number of patients selected for this study was 459: MII-pH recording ( $\mathrm{F}: \mathrm{M}=58: 33$, aged 2-8 years) WB-pH recording ( $\mathrm{F}: \mathrm{M}=223: 145$, aged $2-0$ years).

All patients who undergone the MII-pH method successfully completed the 48-hours recording, whereas, 24/368 $(6.52 \%)$ of patients undertaking the WB-pH study encountered technical faults (capsule detachment sooner or significant artefacts captured) thus requiring to repeat the WB-pH study $(\mathrm{p}=0.0040)$.

IRR of the 2 days of reflux monitoring was significantly higher in patients who underwent MII-pH $(89.0 \%$ vs $69.8 \%$, $\mathrm{p}=0.0018$ ). The diagnostic yield of GORD by extending MII$\mathrm{pH}$ by the second $24-\mathrm{hr}$ study increased by $5.5 \%$ whereas $45.6 \%$ of the patients who underwent WB-pH monitoring showed GORD on day 1 thus not requiring the extended reflux monitoring $(\mathrm{p}<0.0001)$. Only $9.0 \%$ of patients who undertook WB-pH demonstrated pathological reflux on day 2 when normal recording was observed on day 1 .

Conclusion MII-pH monitoring technique seems to offer stronger reliability of diagnosis between 2 consecutive days of recording and seems to be less prone to technical difficulties

\section{REFERENCE}

1. Ayazi S., Lipham J., Portale G., et al. 2009. Bravo catheter-free $\mathrm{H}$ monitoring: normal values, concordance, optimal diagnostic thresholds and accuracy. Clinical gastroenterology and Hepatology, Vol. 7; p6-7

\section{PWE-093 INTER-RATER CORRELATION OF OESOPHAGEAL MOTILITY DURING LIQUID SWALLOW AND SOLID SWALLOW IN PATIENTS WITH ACHALASIA}

Ismail Miah*. Guy's and St Thomas' NHS Trust, Greater London, UK
Introduction Patients attending the oesophageal laboratory mainly complaining of dysphagia are generally to solid food. However current motility assessment to diagnose achalasia and the subtype are based on water swallows [1]. In this study, we investigate the correlation between liquid swallow and solid swallow in patients with achalasia.

Method Patients were selected based Chicago Classification (version 3)[1] between January and December of 2018. Highresolution manometry was performed using the Sierra Scientific Instruments with 36-channel solid-state catheter and Manoscan 3.0.1 was used to capture the recording. Solid bolus swallows were performed using bread with butter sandwich meal and motility assessment (including distal contractile integral values) was based on [2]

Inter-rater reliability (IRR) was calculated between liquid swallow and solid swallow in the achalasia subtypes. Fisher exact was employed to find investigate statistical differences in the IRR between achalasia subtypes during liquid swallow and solid swallow.

Results Patients selected (F:M=45:43, aged $=1-7$ years) were of subtype 1, 2, and 3 respectively of $39.8 \%, 60.2 \%$ and $0 \%$ based on water swallows.

The total number of patients performing liquid and solid bolus swallows in achalasia subtypes 1 and 2 were 29 and 38 .

The inter-rater agreement between liquid swallow and solid swallow in achalasia subtypes 1 and 2 were $37.9 \%$ and 86.8\% ( $\mathrm{p}<0.0001)$.

Conclusion Achalasia type 2 shows strong agreement between liquid bolus swallow and solid bolus swallow.

Majority of type 1 achalasia on solid swallows were not consistent with the primary diagnosis made by liquid swallow.

\section{REFERENCE}

1. Kahrilas PJ, et al. The Chicago Classification of Esophageal Motility Disorders, v3.0 Neurogastroenterol Motil 2015;27(2):160-174.

2. Sweis RT, et al. Normal values and inter-observer agreement for liquid and solid bolus swallows in upright and supine positions as assessed by esophageal highresolution manometry. Neurogastroenterol Motil2011;23(6):509-516.

\section{PWE-094 ACHALASIA SUBTYPE CLASSIFICATION BASED ON LIQUID SWALLOW AND SOLID SWALLOW}

Ismail Miah. Guy's and St Thomas' Nhs Trust, , UK

\subsection{6/gutjnl-2019-BSGAbstracts.414}

Introduction The oesophageal body motility in achalasia defines the subtype of the disease. However, it is unknown how the oesophageal body motility changes when challenged with solid swallows as our clinical practice focuses on water swallows. The aim of this study addresses the topic in question.

Method Patients were selected based on Chicago Classification (CC) (version 3)[1] between January to December 2018. High-resolution manometry was performed using the Sierra Scientific Instruments with 36-channel solid-state catheter and Manoscan 3.0.1 was used to capture the recording. Solid bolus swallows were performed using bread with butter sandwich meal and motility assessment (including distal contractile integral [DCI] values) was based on [2]. Fisher exact test was employed to assess for any statistical difference where appropriate. 
Results Total number of achalasia patients selected was 67 (F: $\mathrm{M}=45: 43$, aged 1-7 years) and achalasia subtypes 1,2 and 3 based on CC were respectively $39.8 \%, 60.2 \%$ and $0 \%$. The solid swallow outcomes for achalasia subtypes 1 and 2 are in table 1 .

$\begin{aligned} & \text { Abstract PWE-094 Table } 1 \\
& \text { challenged with solid bolus }\end{aligned}$
\begin{tabular}{lll}
\hline Water swallows outcome & Type $1(n=29)$ & Type $2(n=38)$ \\
\hline Solid swallows outcome & 11 (type 1) & 1 (type 1) \\
& 15 (type 2) & 38 (type 2) \\
& 3 (peristalsis observed) & 2 (peristalsis observed) \\
\hline
\end{tabular}

In $62.1 \%$ of patients with primary type 1 achalasia diagnosis on liquid swallow have shown inconsistent features on solid bolus swallow ( $>20 \%$ panoesophageal pressurisation or peristalsis) whereas $86.8 \%$ patients with type 2 achalasia diagnosis on primary diagnosis from liquid swallow also showed panoesophageal pressurisation on solid bolus swallows $(p=0.0002)$. There was evidence of peristalsis on solid swallows in type 1 achalasia and type 2 achalasia by $10.2 \%$ and $2.63 \%$ respectively.

Conclusion Type 2 achalasia mostly remains unchanged from liquid swallows to solid swallows. The oesophageal body motility in type 1 achalasia seems to change in $>50 \%$ when challenged with solid bolus. It is unknown which swallow test method offers the best prognosis for achalasia treatment.

\section{REFERENCE}

1. Kahrilas PJ, et al. The Chicago Classification of Esophageal Motility Disorders, v3.0 Neurogastroenterol Motil 2015;27(2):160-174.

2. Sweis RT, et al. Normal values and inter-observer agreement for liquid and solid bolus swallows in upright and supine positions as assessed by esophageal highresolution manometry. Neurogastroenterol Motil2011;23(6):509-516.

\section{PWE-095 SANDIFER SYNDROME: PRESENTATION, INVESTIGATION AND MANAGEMENT}

Irina Mindlina*. University Of Cambridge School Of Clinical Medicine, Cambridge, UK

\subsection{6/gutjnl-2019-BSGAbstracts.415}

Background Sandifer syndrome is a rare complication of gastro-oesophageal reflux disease (GORD) when a patient presents with extraoesophageal symptoms, typically neurological. Although its pathophysiology is not completely understood, one explanation is that neurological manifestations are the consequence of vagal reflex with the reflex centre in nucleus tractus solitarii. The goal of this study was to review the existing literature and describe a typical presentation and most appropriate investigations and management for the Sandifer syndrome.

Methods A comprehensive literature search was performed via PubMed, Cochrane Library and NHS Evidence databases. 27 cases and observational studies were identified.

Results The literature demonstrates that presenting symptoms of Sandifer's may include any combination of abnormal movements and/or positioning of head, neck, trunk and upper limbs, seizure-like episodes, ocular symptoms, irritability, developmental and growth delay, iron-deficiency anaemia.

In terms of diagnostic procedures, 24-hour oesophageal $\mathrm{pH}$ monitoring was positive in all the cases of Sandifer's where it was performed, while upper GI endoscopy +/- biopsy and barium swallow were diagnostic only in a subset of cases.

A range of treatment options were applied in the reviewed literature, including dietary changes, pharmacological management, enteral tube feeding, and surgical approach. These treatment options are consistent with the 2015 NICE guidelines on the management of GORD in children and young people.

Successful treatment of the underlying gastro-oesophageal pathology led to a complete or near-complete resolution of the neurological symptoms in all of the cases.

Discussion As Sandifer syndrome is driven by the underlying gastro-oesophageal reflux, it is not surprising that its investigations and management showed to be consistent with those of GORD. It is evident from the literature that many patients were originally misdiagnosed with various neuropsychiatric diagnoses that led to unnecessary testing and ineffective medications with significant side effects. Earlier diagnosis of Sandifer's would have allowed to avoid them.

\section{PWE-096 RECTAL HYPERSENSITIVITY IN IBS: A SYSTEMATIC REVIEW AND META-ANLAYSIS}

Christopher Roberts*, Ahmed Albusoda, Qasim Aziz. Wingate Institute of Neurogastroenterology, Queen Mary, London, UK

\subsection{6/gutjnl-2019-BSGAbstracts.416}

Introduction Rectal hypersensitivity is defined as being an exaggerated response to painful rectal stimuli and is thought to occur in Irritable Bowel Syndrome (IBS) although it is not a universal observation. The aim of this systematic review and meta-analysis is to confirm the presence and determine how common rectal hypersensitivity is in IBS and to determine if there are other factors such as IBS criteria, age, gender and IBS subtype that affect the presence of rectal hypersensitivity. Secondary aims were to see if rectal hypersensitivity was present in IBD to see if inflammation plays a key role in rectal hypersensitivity hence a second meta-analysis was performing assessing rectal hypersensitivity in IBD.

Methods A systematic review of the literature from MEDLINE and EMBASE databases (1970-Present) was carried out. Only prospective studies were included that compared pain or discomfort thresholds to mechanical rectal stimuli in an IBS and a control population. The second review looked at studies that measured pain or discomfort thresholds to mechanical rectal stimuli in an IBD and a control population. Data was pooled for both meta-analyses and Hedges $\mathrm{g}$ effect sizes were calculated with 95\% confidence intervals (CIs). This review is registered with PROSPERO (CRD42018095687).

Results The search strategy identified 684 studies of which 70 were deemed relevant and 31 studies met the inclusion criteria. Rectal sensitivity was greater in an IBS population compared to controls. The effect size calculated was $0.96695 \%$ CIs $(0.76-.166) \quad(p<0.0001)$. Significant heterogeneity was seen $\left(\mathrm{I}^{2}=77.2 \%\right)$. Secondary analysis revealed that gender, IBS subtype and IBS criteria did not have a significant impact upon the presence of rectal hypersensitivity. When comparing adult and paediatric IBS populations there was a significant difference. There was no statistically significant difference between IBD and healthy controls 0.456 95\% CIs $(-0.20-$ $.119)(\mathrm{p}=0.178)\left(\mathrm{I}^{2}=87.6 \%\right)$. However, a significant difference when comparing those with active flares of their IBD against those studies that used quiescent IBD was found. 